\title{
Invited review: Genetic contributions underlying the development of preimplantation bovine embryos
}

\author{
J. Kropp, ${ }^{*}$ F. Peñagaricano, ${ }^{*}$ S. M. Salih, $\dagger$ and H. Khatib*1 \\ *Department of Animal Sciences, and \\ †Department of Obstetrics and Gynecology, University of Wisconsin, Madison 53706
}

\section{ABSTRACT}

In recent years, it has become evident that genetic selection to improve milk production has resulted in a decline in dairy cattle fertility. Growing evidence suggests that the greatest loss occurs early in pregnancy around the time of embryo implantation. As a means to make genetic improvements and to assist in reproductive performance, use of artificial reproductive technologies such as artificial insemination and in vitro production of embryos have been widely used. Both of these technologies rely on the competence and quality of gametes for successful development of embryos. Often, selection of animals is based on the genetic merit of the animal, although specific fertility markers are relatively underdeveloped compared with markers for production traits. Similarly, current in vitro fertilization systems could benefit from a uniform method for selection of the best quality embryos to transfer into recipients for successful implantation and delivery of healthy offspring. As genetics underlie biological processes such as fertility, the need exists to further identify and characterize genes that affect fertility and development within both the parental gametes and the embryo. Furthermore, the magnitude of the contribution of each parental genome to the success of embryo development and pregnancy is not clear. As such, the objective of this review is to provide an overview of studies relating to genetic markers at the DNA level, parental and embryonic gene expression, and the effects of epigenetics on embryonic development. Future studies should exploit advances in molecular technologies to identify and classify genes underlying fertility and development to establish biomarkers and predictors for improved genetic selection.

Key words: embryo, in vitro fertilization, epigenetics, gene expression

Received July 9, 2013.

Accepted November 8, 2013.

${ }^{1}$ Corresponding author: hkhatib@wisc.edu

\section{INTRODUCTION}

Over the last $40 \mathrm{yr}$, genetic improvements have met the demanding needs for greater milk production; however, the relative fertility of dairy cattle has decreased. With high demand for agricultural products, assisted reproductive technologies have been used to better cope with the economic pressure and the decreasing fertility. The dairy cattle fertilization rate is about $80 \%$; however, only about 30 to $50 \%$ of dairy cows inseminated successfully calve (Evans and Walsh, 2011). Furthermore, the greatest degree of embryonic loss occurs before d 16, with even greater loss before d 8 in the high-producing dairy cow (Diskin et al., 2011). In vitro production of embryos has been used not only as a method to generate offspring of elite producing cattle, but also as a genetic model for studying reproductive systems (Moore and Thatcher, 2006). In an in vitro system, the proportion of embryos that develop to the blastocyst stage is about 20 to $50 \%$ (Rizos et al., 2002a,c; Moore and Thatcher, 2006). Improvements in understanding the genetics of gametes and early preimplantation embryos could help combat the low efficiency and high embryonic loss observed within these production systems.

Currently, selection of preimplantation mammalian embryos is most often based on morphological assessment following standards set by the International Embryo Transfer Society (Van Soom et al., 2003). Morphological assessment is composed of visual assessment of cell number, fragmentation, compaction, and color for grading the embryo in terms of stage and quality. Grading criteria are in concordance with the standards set by the International Embryo Transfer Society (Van Soom et al., 2003). Although embryos may be of similar morphology, they may not have similar developmental competence. It is known that in vivo-derived bovine embryos develop better than similar-appearing in vitro-derived embryos (Crosier et al., 2001; Rizos et al., 2002a,b,c; Van Soom et al., 2003). Ultrastructural morphometric analysis of in vivo- versus in vitro-derived blastocysts has revealed that in vitro blastocysts have increased cytoplasm-to-nuclear ratios, increased lipid 
volume, and decreased total mitochondria volume compared with in vivo blastocysts (Crosier et al., 2001). Although close morphological assessment can distinguish embryos based on these physical properties, it does not reflect the genetic composition and developmental potential of the embryo.

A more efficient method of embryo selection would assess the potential reproductive success of gametes and embryos based on their underlying genetic potential. Genomic studies should not be limited to the embryo's genome but rather extended to the parental contributors from which its genome is derived. Development of biological markers as predictors in both parental gametes and the embryo itself for successful outcomes could greatly improve efficiency of reproduction strategies. As gains have been made in selecting for milk production traits, the adverse effects on fertility may in part be a result of parental genotype on the offspring.

Several strategies have been used in investigating the genetic components of fertility and subsequent embryonic development. Potential predictors can be derived from the study of the genotypic, transcriptomic, and epigenetic profile of both the parental gametes and the developing embryo. Early genomic strategies include the identification of parental genotypes as predictors using genome-wide association studies (GWAS) (Feugang et al., 2009; Huang et al., 2010b; Sahana et al., 2010; Cole et al., 2011; Berry et al., 2012; Peñagaricano et al., 2012a), the candidate gene approach (Russell et al., 2006; Racedo et al., 2008; Pretheeban et al., 2009; Racedo et al., 2009; Arangasamy et al., 2011; Kepkova et al., 2011; Laporta et al., 2011; Zhang et al., 2011; Kasimanickam et al., 2012; O'Doherty et al. 2012), and candidate pathway analysis (Khatib et al., 2009, 2010; Laporta et al., 2011; Fortes et al., 2012; Li et al., 2012a; Peñagaricano et al., 2012b). More recently, a shift to studying the transcriptome has been advantageous for the characterization of gamete-specific transcripts as well as those genes expressed in a stage-specific manner throughout embryonic development (Memili and First, 2000; Misirlioglu et al., 2006; Kues et al., 2008; Lalancette et al., 2008; Racedo et al., 2008; Katz-Jaffe et al., 2009; Tesfaye et al., 2009; Huang et al., 2010b; Arangasamy et al., 2011; Mamo et al., 2011; Robert et al., 2011; Driver et al., 2012; Gad et al., 2012; Govindaraju et al., 2012; Kasimanickam et al., 2012; Abd El Naby et al., 2013; Card et al., 2013). Evolution of technology from microarrays to next-generation sequencing, in combination with quantitative real-time PCR (qPCR), has allowed for identification of novel and lowly expressed genes that may be relevant to the prediction of the genetic potential of the gamete or the embryo.
Recent progress in identifying predictors of development lies within the growing field of epigenetics. Epigenetics refers to heritable changes in gene expression without changes in the DNA sequence itself (Bressan et al., 2009). Epigenetic modifications control the regulation of many genes associated with fetal development and placental growth (Bressan et al., 2009; Kono, 2009; Obata, 2011). Appropriate reprogramming of these modifications may be of crucial importance to embryonic development and sustaining pregnancy; thus, growth of knowledge in this area could contribute greatly to understanding gene expression mediating biological processes.

In vitro production systems have been widely used to better study gametes and embryonic development. These systems have allowed for generation of a large number of progeny from selected gametes with minimal variation in the production system environment. However, the in vitro production environment imposes stresses that are unnatural to an embryo, which may potentially alter gene expression in itself. Studies have indicated that in vitro embryos differ from in vivo-derived embryos in terms of having higher lipid content, fewer mitochondria, fewer cellular divisions, and different gene expression (Crosier et al., 2001; Rizos et al., 2002a,b; Driver et al., 2012). A better understanding of developmental mechanisms altered by the production environment could aid in the development of predictors to indicate which embryos may develop successfully within a given system.

Former reviews critically analyze the paradigm of an increase in production versus the decline in reproduction in cattle but are often sex-specific and do not include both genetic parental contributions to the embryo. The focus of these reviews illustrates animal health components, estrus detection, reproductive management programs, and energy balance (Lucy, 2001; Leroy et al., 2008; Diskin et al., 2011; Evans and Walsh, 2011; Walsh et al., 2011). The review by Veerkamp and Beerda (2007) aimed to examine genomics of fertility and genomic selection for the improvement of fertility focused on functional genomic studies for fertility. However, in the current age of systems biology and "omics," a review that combines genomics, transcriptomics, proteomics, and epigenetic contributions to the field would bring greater perspective to future research aims. Therefore, the aim of this review is to discuss the current bovinespecific data from varying research approaches to better illustrate the genetic architecture of the fertility of both the dam and sire contributing to embryonic development. Although in vitro production systems have the potential of altering expression, they have proven to be a highly effective research tool. Inclusion of both in 
vivo and in vitro systems can be used to better understand the underlying genetics, not only of the parental gamete but also of early embryonic development. Additionally, different environmental factors have recently been shown to affect epigenetic mechanisms within both gametes and embryos and may greatly affect early and later embryonic development. Although many current studies are often hypothesis-generating studies, here a comprehensive review of specific markers, including differentially expressed genes in varying populations, gene relationships, and epigenetic markers, will be outlined to better understand the biology underlying dairy cattle reproduction. In the following sections, aspects of both male and female fertility will be discussed at the genomic, transcriptomic, and epigenomic levels. In addition, the embryonic transcriptome and epigenome will be reviewed to better understand the roles that parental contributions and activation of the embryo's own genome have on the progression through development. As fertility declines, the generation of biomarkers or predictors for both gamete and embryo selection may allow for improvements in embryonic development and, subsequently, higher fertility and healthier offspring.

\section{FEMALE AND MALE FERTILITY AT THE GENOMIC LEVEL}

Initial research to identify the genetic relationships underlying fertility decline largely focused on differences at the genomic level, where the greatest contributions have mainly centered on female fertility versus male fertility. Indeed, some recent studies have attempted to identify genomic regions associated with female fertility using high-density markers across the genome and fertility measures typically recorded in the field, such as days to first service, pregnancy rate, number of services, and calving interval. For instance, Sahana et al. (2010) conducted a whole-genome study for 11 fertility traits in Danish and Swedish Holstein cattle using data from 2,531 progeny-tested bulls and a total of 36,387 SNP across the genome. A total of 24 significant genomic regions on 14 chromosomes were detected. The authors argued that these regions would facilitate searches for candidate genes and polymorphisms associated with cow fertility. In another study, Cole et al. (2011) carried out a genome-wide association analysis for 31 production, health, reproduction, and body conformation traits in US Holstein cows. In particular, these authors reported significant associations between genomic regions on Bos taurus chromosome 3 (BTA3), BTA7, and BTAX and daughter pregnancy rate (DPR) as a measure of female fertility. Specifically, the authors proposed INSR, $A T P 1 B 4$, and GRIA3 as candidate genes highly related to this trait. In particular, ATP1B4 encodes a protein that may interact downstream in regulating transforming growth factor (TGF)- $\beta$ signaling and, similarly, GRIA3 is a receptor involved in neurotransmission (Cole et al., 2011). Furthermore, Berry et al. (2012) reported results from a genome-wide study for fertility traits in Holstein cows using data from experimental research herds in 4 European countries. Interestingly, the authors analyzed traditional measures of fertility, as well as a fertility phenotype derived from milk progesterone profiles. Several genomic regions were found to be associated with different female fertility traits, although they only explained a small proportion of the total genetic variance in the traits (Berry et al., 2012).

Recently, Cochran et al. (2013a) evaluated the association between SNP in 434 genes closely related to reproductive processes and fertility and production traits in Holstein cattle. In particular, 40 genes were significantly associated with DPR. Among these were genes involved in cell signaling, inhibition of apoptosis, and endocrine and immune systems. As expected, some significant SNP positively associated with DPR were negatively associated with production traits. Given that several SNP associated with DPR did not show association with production traits, the authors concluded that it should be possible to select for DPR without compromising production in dairy cattle.

However, identification of specific genetic markers or even genes related to fertility traits has been a challenging task, partly due to the complex nature of these traits (e.g., poorly defined phenotypes) and their low heritabilities (Veerkamp and Beerda, 2007). Although many factors account for the decline in reproductive performance of dairy cows, the most important component seems to be a reduction in fertilization and embryonic survival rates (Santos et al., 2004). With this in mind, a bovine in vitro fertilization (IVF) system was established in our laboratory to recapitulate early embryonic development up to the blastocyst stage. Two important components of fertility, fertilization rate and blastocyst rate, were recorded. As embryos in this IVF system are cultured in a unified environment, variation due to environment is minimized, and thus the power to detect genetic markers increases. In fact, using this system, several pathways and single genes have been found to be associated with IVF fertility traits (Khatib et al., 2008a,b, 2009; Driver et al., 2009; Peñagaricano and Khatib, 2012). For instance, Khatib et al. (2009) evaluated the possible involvement of the POU1F1 pathway in early embryo development. Genes within this pathway have functions in signal transduction pathways associated with milk production traits. These authors reported significant associations of GHR, PRLR, STAT5A, and UTMP with blastocyst rate and of POU1F1, GHR, STAT5A, and OPN with fertiliza- 
tion rate. Interestingly, adjacent genes in the pathway showed significant interaction effects, denoting that polymorphisms in these genes as well as gene interactions are important in determining the phenotypic variation in fertilization and blastocyst rates. Interestingly, some genotypes were identified with an antagonist relationship between reproduction and production traits. For instance, SNP in STAT5A, GHR, PRLR, and $U T M P$ genes were positively associated with IVF traits and negatively associated with milk yield and fat and protein production (Khatib et al., 2009).

Using our IVF experiment system, Huang et al. (2010a) extended the mapping effort utilizing a highdensity SNP genotyping array and a selective DNA pooling approach. In this study, 22 and 5 SNP were found to be associated with fertilization rate and blastocyst rate, respectively (Huang et al., 2010a). For instance, one SNP associated with fertilization rate is within an intron of E2F2, a member of the E2F transcription factor family that controls progression of the cell cycle. Importantly, loss of E2F2 in Drosophila results in partial female sterility due to changes in DNA replication in follicle cells (Huang et al., 2010a). The authors concluded that these significant SNP could be used in future studies to select candidate genes for resequencing and fine mapping.

Recently, Cochran et al. (2013b) evaluated the possible association between several candidate genes and fertilization and early embryonic development using an analogous IVF system. Nine genes were associated with cleavage rate, and 12 genes were associated with blastocyst development rate. That study provides further evidence that IVF traits are under genetic control and supplies a new list of genes with potential roles in these important processes. Interestingly, the study could not validate previous candidate genes; for example, $P G R$, STAT5A, and FGF2. As explained by Cochran et al. (2013b), different IVF measurements and different genotyping strategies, among other factors, could explain the lack of repeatability.

In general terms, bull fertility has received much less consideration compared with cow fertility. Nevertheless, some studies have reported that a significant percentage of reproductive failure in dairy cattle is attributable to male subfertility (DeJarnette et al., 2004). Consequently, bull fertility should not be overlooked in the quest to improve the reproductive performance of dairy cattle (Braundmeier and Miller, 2001). Indeed, there is growing evidence that bull fertility is influenced by genetic factors. Using a candidate pathway approach, Khatib et al. (2010) reported that polymorphisms in FGF2 and STAT5A, 2 genes located in the POU1F1 signaling pathway, are associated with estimated relative conception rate in Holstein bulls. The STAT5a gene, whose protein functions within a signal transduction pathway including IFN- $\tau$ signaling for maternal recognition of pregnancy, was found to be expressed in oocytes as well as blastocysts. Using a marker within the genes in the study by Khatib et al. (2009), it was found that that monoallelic expression of STAT5a was significantly higher in blastocysts, with greater paternal expression in degenerate embryos (those that fail to transition from the morula to blastocyst stage). Similarly, Lan et al. (2013) found that a missense mutation in PROP1, a regulatory gene of the POU1F1pathway, is also associated with bull fertility in dairy cattle. These findings suggest that genetic polymorphisms in the PROP1/ POU1F1 pathway could be used in selection programs to improve male fertility in cattle.

Furthermore, a comparative genomics study evaluated the association between spermatogenesis genes highly conserved from fly to human and sire conception rate (SCR) in US Holsteins bulls (Li et al., 2012b). Interestingly, $3 \mathrm{SNP}$ located in $M A P 1 B$ and $1 \mathrm{SNP}$ in PPP1R11 showed significant associations with SCR (Li et al., 2012b). Of the most significant SNP, 3 were found within the $M A P 1 B$ gene; $M A P 1 B$ (microtubule-associated protein $1 \mathrm{~B}$ ) is a gene associated with spermatogenesis and is involved in the interaction between actin microfilaments and microtubules ( $\mathrm{Li}$ et al., 2012b). The other gene found to have significant SNP association with SCR, PPP1R11, was previously identified to be associated with spermatogenesis and motility in mice and humans but here is the first association with bovine sire fertility. These findings testify to the usefulness of the comparative genomics approach in selecting candidate male fertility genes.

Recently, some studies have addressed bull fertility using a genome-wide association approach. For instance, Feugang et al. (2009), using a 2-stage GWAS, showed that the ITGB5 (integrin $\beta$ 5) gene is involved in the fertilization process in Holstein bulls; ITGB5 is thought to play a role in cell-cell interaction as well as potentially serve as a signaling receptor at the time of fertilization (Feugang et al., 2009). In addition, Blaschek et al. (2011) identified several genomic regions associated with noncompensatory bull fertility. Fortes et al. (2012) reported that genomic regions in BTA2, BTA14, and BTAX are associated with testicular development, sperm quality, and different hormone levels measured throughout puberty in Brahman bulls. Furthermore, Peñagaricano et al. (2012a) identified a panel of SNP associated with SCR in US Holstein sires. Some of the significant SNP were located within or near genes affecting male fertility, such as the sperm acrosome reaction, chromatin remodeling during spermatogenesis, or the meiotic process during male germ cell maturation (Peñagaricano et al., 2012a). Altogether, these studies 
have identified genomic regions and individual genes associated with bull fertility.

Genome-wide association studies typically focus on genetic markers with the strongest evidence of association. However, these markers explain only a small component of the genetic variance and hence offer an incomplete understanding of the trait under study. By taking another approach, Peñagaricano et al. (2012b) performed a pathway-based association analysis in a large Holstein bull population to attempt to identify relevant pathways involved in bull fertility. Twenty Gene Ontology (http://www.geneontology.org/) categories and one InterPro (http://www.ebi.ac.uk/interpro) entry showed significant overrepresentation of genes statistically associated with bull fertility. Several of these functional categories such as small GTPasemediated signal transduction, neurogenesis, calcium ion binding, and cytoskeleton are known to be involved in biological processes closely related to male fertility (Peñagaricano et al., 2012b). Importantly, that study provides evidence that the quantitative trait pathway approach could complement GWAS for investigating quantitative traits in livestock species.

Overall, these studies have identified genomic regions, candidate genes, and candidate pathways associated with female and male fertility at the genomic level. All of these studies contribute to a better understanding of the genetics underlying complex traits in cattle. In addition, this information could be used in markerassisted selection in future cattle breeding schemes.

\section{TRANSCRIPTOMICS OF FERTILITY AND DEVELOPMENT}

\section{Maternal Gene Expression}

Early research has found that stored within the oocyte are RNA molecules and proteins that greatly contribute to early embryonic development (Memili and First, 2000). Oocyte competency refers to the ability of the oocyte to undergo maturation and subsequent fertilization. Before fertilization, it is necessary for the oocyte to undergo both meiotic and cytoplasmic maturation, where these events contribute to oocyte competency. Meiotic maturation or nuclear maturation within the oocyte occurs through either induction by a luteinizing hormone ( $\mathrm{LH})$ surge at ovulation or physical removal of the oocyte from the follicle (Sirard et al., 2006). Physiologically, the nuclear component of the oocyte will undergo resumption of meiosis and progression from meiosis I to meiosis II. This event can be morphologically characterized by the extrusion of the first polar body and the presence of a metaphase plate. Whereas meiotic maturation is observable through microscopy, cytoplasmic maturation results in the redistribution of cortical granules and organelles and is difficult to assess (Sirard et al., 2006; Mamo et al., 2011). Oocyte competency is believed to dictate the earliest events leading up to embryonic genome transcription activation through the stored products of oogenesis and maturation. Investigation into stored maternal gene products has led to the characterization of specific transcripts within the oocyte. An early study by Racedo et al. (2008) sought to test the expression of known transcripts within oocytes derived from follicles of varying size and maturation stage. It was found that oocytes from 2- to 8-mm follicles - which have proven to develop better than oocytes from $<2$-mm folliclesdiffered in the relative abundance of transcripts for genes with functional relevance in oocyte maturation. In addition, genes encoding motor proteins were not only more abundant in immature oocytes from larger 2 - to 8-mm follicle-derived oocytes, but also the relative abundance varied across stages of maturation for the 2 different follicle sizes (Racedo et al., 2008). Thus, evidence shows that abundance of certain transcripts with important roles in maturation depends on follicle size and stage of maturity. In attempts to better characterize oocyte quality, Mamo et al. (2011) used a microarray strategy to identify 2,117 transcripts that were differentially expressed between mature and immature in vitro-derived oocytes. Genes overexpressed in matured oocytes were associated with functions in cellular processes and communication, whereas underexpressed genes were associated with metabolic processes. In this same experiment, the addition of a transcription inhibitor, $\alpha$-amanitin, resulted in overexpression of 25 genes tested in the in vitro-matured group. Expression in the in vitro-matured group mimicked the expression of the immature oocytes as a result of the inhibitor. These studies illustrate that dynamic changes in the oocyte transcriptome during maturation may contribute to oocyte competency.

Another approach has focused on the roles of oocytespecific transcripts in early development. Kues et al. (2008) used microarrays to study transcripts in both oocytes and embryos of various stages. The study found 35 genes specifically upregulated in oocytes compared with embryos of varying stages of development. Further expression analysis of 6 of the genes upregulated in oocytes revealed that, following fertilization, the expression of these transcripts declined. Thus, this hypothesis-generating study identified oocyte-specific transcripts that are more important for early embryonic development rather than later stages of development.

Oocytes matured in vivo have higher development rates than those matured in vitro; therefore, transcripts produced in different maturation environments have 
been a focus of many studies. By analyzing various types of maturation media and supplements used in in vitro production, as illustrated by Russell et al. (2006), it was found that key genes were differentially expressed in blastocysts derived from oocytes of varying maturation environments. These genes included $I F N \tau$, Oct-4, IGFBP6, and HDacl, which function to regulate other gene products through signaling, transcription, and organization of chromatin. By analyzing different media components, differential expression of a panel of genes was observed by altering the media composition (Russell et al., 2006). Further analysis of production environment on embryonic development has been achieved by comparing expression of in vivo- versus in vitro-matured oocytes. Kues et al. (2008) reported that 1,799 transcripts were differentially expressed between in vivo and in vitro maturation conditions. In addition, in vivo oocytes had a greater number of transcripts in total $(12,049)$ compared with in vitro oocytes $(11,332)$. Similarly, Katz-Jaffe et al. (2009) analyzed transcripts of oocytes derived from in vivo or in vitro maturation and reported that 10 genes were differentially expressed. Gene ontology analysis showed that genes relating to metabolism, such as those with roles in the tricarboxylic acid cycle and oxidative phosphorylation, were downregulated in in vitro-matured oocytes (Katz-Jaffe et al., 2009). The oocyte maturation environment thus contributes to differences in gene products particularly relating to metabolism and therefore a difference in stored products within the oocyte.

More recently, a newly identified class of RNA molecules that act to regulate the transcript abundance within the oocyte has been studied. MicroRNAs (miRNA) have been studied within immature and mature oocytes due to their function in regulating mRNA posttranscriptionally. In a study by Tesfaye et al. (2009), a heterologous microarray composed of probes from human, mouse, and rats was used to profile the miRNA within oocytes of varying stages. A total of 59 miRNA were differentially expressed, and 32 of these miRNA were previously unidentified in bovine. It was found that 31 miRNA were preferentially expressed within immature oocytes, whereas 28 miRNA were more abundant in matured oocytes. Comparative array strategies have provided a novel approach to identify miRNA in the bovine; however, future research should progress to identify their function on the genes that they regulate. As seen in the study by Tesfaye et al. (2009), stage-specific expression of these miRNA may be a function of temporal regulation of certain transcripts within the oocyte.

A study by Abd El Naby et al. (2013) further investigated the role of miRNA within the oocyte and surrounding cumulus cells supporting the oocyte. It was found that 8 miRNA were enriched in cumulus cells of immature oocytes and 39 were enriched in immature oocytes, with fold changes in expression ranging from 56 to 2,109. Similarly, 6 miRNA were enriched in cumulus cells of mature oocytes and 45 were enriched in mature oocytes with fold changes in expression ranging from 109 to 2,433. Through bioinformatics, the function of genes targeted by differentially expressed miRNA could be analyzed. This approach identified 25 and 13 molecular networks that contained the miRNA target genes enriched in oocytes and cumulus cells, respectively. These networks include many genes relating to cellular development and signaling processes (Abd El Naby et al., 2013). Interactions between the oocyte and cumulus cells add further complexity to understanding the dynamic changes in gene expression to mature a competent oocyte. Taking into account the regulatory function of miRNA, differentially expressed miRNA during oocyte maturation may dictate the presence and abundance of certain transcripts stored within the oocyte before fertilization.

\section{Paternal Gene Expression}

Previously, less emphasis has been placed on the study of sperm because of the assumption that they are transcriptionally inactive. Although transcriptional activity decreases as spermatocytes progress through the meiotic divisions of spermatogenesis, the presence of different classes of RNA such as mRNA, miRNA, and noncoding RNA (ncRNA) in mature sperm is well known (Lalancette et al., 2008; Ogorevc et al., 2011; Kasimanickam et al., 2012). Ostermeier et al. (2004) concluded from human spermatozoa-hamster sperm hybrids that certain transcripts, such as protamine 2 and clusterin, were specific to spermatozoa and zygotes and absent in oocytes, suggesting sperm-specific delivery of RNA at the time of fertilization. More recently, it has been found that sperm contribute at fertilization about 5,000 RNA, of which $25 \%$ encode for proteins regulating transcription and transcription factors (Arangasamy et al., 2011). Further development and characterization of markers of sperm quality and stored products delivered to the oocyte may expand our understanding of successful fertilization and subsequent embryonic development. An approach used to assess paternal contribution has been to study the relationship of sperm transcripts from bulls of a high or low field fertility measure. An early study by Lalancette et al. (2008) profiled the transcriptome of sperm of bulls with high and low nonreturn rates using suppressionsubtractive hybridization. Generation of transcript libraries resulted in $29 \%$ of the high-nonreturn rate library associated with known functions such as me- 
tabolism and signal transduction, whereas only $10 \%$ of low-nonreturn rate transcripts were associated with known functions. Elucidating the transcripts specific to high- or low-fertility bulls may lead to a better understanding of the mechanisms causing reduced fertility.

Another method, adapted by Arangasamy et al. (2011) and subsequently used by Kasimanickam et al. (2012), allows for the study of relative mRNA abundance of candidate sperm-specific proteins in extreme high or low SCR bulls. Association of given candidate sperm-related proteins with a given field fertility measure, such as SCR, enables the study of transcript abundance associated with a phenotype or function of sperm proteins. In the early study by Arangasamy et al. (2011), the genes CRISP2 and PEPB1, which have functions in sperm capacitation and sperm-egg interactions, were both more highly expressed in positive SCR bulls. In the multivariate regression model used, it was found that these genes explained $95 \%$ of the variance in the SCR scores of the 34 Holstein bulls tested. An interaction was also observed between these genes, although the exact interaction is to be determined. In future analysis, considerations should similarly focus on gene interactions and characterization of gene functions and pathways for a more thorough understanding of the underlying biologic mechanisms to explain differences observed in bull fertility.

In a subsequent study, the expression of 9 spermspecific protein genes was tested for an association with SCR, where key genes in signaling and growth, as well as an imprinted gene, were included (Kasimanickam et al., 2012). Interestingly, the genes adenylate kinase, integrin $\beta$, Doppel, nerve growth factor, tissue inhibitors of metalloproteinases, small nuclear ribonucleotide polypeptide N $(S N R P N)$, and PLC-zeta were found to be more abundantly expressed in bulls of high SCR. Interestingly, these genes have biological roles in spermegg interaction, capacitation, acrosome reaction, and motility. Moreover, the imprinted gene $S N R P N$ was found to be abundantly expressed in high SCR bulls, which may be a result of a higher degree of condensation of the DNA by protamines and perhaps an unaltered methylation pattern, indicating an epigenetic effect between high- and low-SCR bulls (Kasimanickam et al., 2012). These studies illustrate that a candidate gene approach and a subsequent association analysis with a field fertility measure may not only aid in the development of biomarkers, but will also classify the function of sperm transcripts for a given field fertility measure.

Another approach to investigate spermatozoa products is by comparison of field fertility with protein expression, as undertaken in the study by Peddinti et al. (2008). This study reported 125 proteins that were differentially expressed between bulls of high and low fertility, of which 74 had increased expression and 51 had decreased expression. Specific functions of these proteins were unique to fertility, with higher fertility bulls exhibiting a greater abundance of proteins related to cellular movement and signaling compared with lower fertility bulls, reflecting proteins related to cellular death. Although a limitation of this approach is that it does not adequately define roles of embryonic development based on proteins, its strength is that it could provide potential biomarkers for sperm selection.

Different classes of RNA are also under study for the relationship of abundance and fertility. Recently, a study by Govindaraju et al. (2012) analyzed miRNA in bull spermatozoa with a field fertility measure. Results of this study revealed higher expression of miRNA in lower fertility bulls. The authors concluded that higher expression could act to downregulate expression of certain genes, potentially including fertility-related genes. Due to the nature of this RNA, it is necessary to not only discover the miRNA within sperm cells, but also identify the target mRNA that they act to regulate. Similar to the female gamete, future work should focus on how miRNA may affect the composition and abundance of transcripts within spermatozoa to alter fertility.

More recently, a global profile of the bovine spermatozoa transcriptome was analyzed. Card et al. (2013) reported the first use of RNA sequencing (RNA-Seq) technology to classify bovine spermatozoa transcripts. The RNA-Seq analysis of a pooled sample of 9 bulls ranging in SCR revealed that both degraded and fulllength transcripts are present in spermatozoa, where full-length transcripts represented $66 \%$ of the transcripts present. Overall function of the full-length transcripts relates to translation with a high abundance of ribosomal proteins and eukaryotic translation initiation factors. Further analysis revealed the presence of both known spermatozoal transcripts confirmed in other species as well as new transcripts previously unidentified in bovine spermatozoa (Card et al., 2013). The authors infer that future work utilizing this technology could be beneficial for classifying transcripts for varying degrees of fertility and thus a potential diagnostic tool for gamete selection in bovine and other mammalian species.

\section{Embryonic Gene Expression}

Preimplantation embryos must surpass many physiologic and morphologic hurdles to complete their development. Early developmental stages include the timing and progression through the first and subsequent cellular divisions, formation of necessary gap junctions, embryonic genome activation (EGA), compaction, 
blastocoel formation, differentiation of cell types, and secretion of embryonic gene products for maternal recognition. It remains unclear whether certain molecular signals driving these early developmental stages are a result of parental contribution or of the embryo itself. Memili and First (2000) reported that bovine zygotic transcription begins as early as the 1-cell zygote stage, termed minor EGA, with a marked increase in transcriptional activity at the 8-cell stage during the maternal embryonic transition (MET), also known as EGA. As the embryo develops, certain genes are turned on in a stage-specific manner; however, the regulation of these genes and the expression levels required for developmental milestones is as yet unclear.

As early development is driven by transcripts delivered to the embryo at the time of fertilization, expression studies have focused on understanding maternalversus embryonic-specific transcripts leading up to EGA. Early work by Misirlioglu et al. (2006) tried to classify oocyte-specific transcripts through the use of $\alpha$-amanitin to block mRNA synthesis, thereby allowing for comparison of transcripts within metaphase-II (MII) oocytes, treated 8-cell embryos, and untreated 8-cell embryos. Via microarray analysis, it was found that 147 genes increased expression by at least 2-fold in 8-cell embryos compared with MII oocytes. Genes within MII oocytes exhibited more diverse functions, including transcription but also DNA methylation, cell adhesion, apoptosis, and metabolism. This evidence establishes that there are maternal-specific transcripts within the oocyte, and as the embryo reaches the 8-cell stage, corresponding to the onset of EGA, the expression of genes relating to transcription increases.

Further evidence was provided by Kues et al. (2008), where the goal was to classify transcript abundance through the different stages of development using microarray analysis. The greatest difference in transcript abundance was between earlier and later stages of development. More specifically, the greatest number of transcripts corresponded to the 2- to 4-cell stage with fewer transcripts from the 4- to 8-cell stage. The authors concluded that the observed decrease was due to rapid rather than gradual degradation of maternal transcripts during maternal embryonic transition. Moreover, it was found that 350 genes were stage-specific, with about 70 total genes more highly expressed in the 2-cell stage that were then downregulated in subsequent stages (Kues et al., 2008). Additionally, this study also classified the genes expressed at the 4- to 8-cell stage, which showed high metabolic and signaling activity, providing evidence of the dynamic changes occurring within the embryo leading up to EGA.

Although it is known that the embryo needs to undergo EGA for successful progression in development, the mechanisms and underlying genetics for development from the morula to blastocyst stage is even more unclear. Huang et al. (2010b) found by microarray analysis that more than 33 genes showed at least a 2 -fold difference in expression between in vitro-derived blastocysts and degenerates. Gene ontology analysis of the genes upregulated in the degenerate embryo population revealed that although many pathways were enriched, one of particular interest was the TGF- $\beta$ pathway. Genes of this pathway have roles in cell division, mitosis, differentiation, and apoptosis. Further analysis of gene expression of the TGF- $\beta$ pathway found that 10 out of 14 genes showed significant differential expression between blastocysts and degenerates; all 10 were upregulated in the degenerate population ( $\mathrm{Li}$ et al., 2012a).

An interesting area of study is the effect of maternal genotype on embryonic gene expression and particularly the alternative splicing of these genes. As reported by Laporta et al. (2011), many genes of the POU1F1 pathway were expressed at a low level in blastocyst and degenerate embryos. The $O P N$ and STAT5A genes, known to be associated with both production and reproduction traits, were found to be upregulated in degenerate embryos compared with blastocysts. Additionally, this study tested the expression of splice variants of the STAT5A gene and found differential expression of transcript 794 between blastocysts and degenerative embryos. Laporta and colleagues (2011) also concluded that both maternal and embryo genotypes affect embryo development. Moreover, critical assessment of alternatively spliced variants should be incorporated into aims of future studies for classification of a variant's biological role.

Another class of genes identified in early embryonic development includes members of the heat-shock protein (HSP) family (Driver and Khatib, 2013). The HSP genes are expressed early during development and are known to function as molecular chaperones to assist in protein folding, transport, and stability. Zhang et al. (2011) compared the expression of 17 HSP genes in blastocysts and degenerate embryos. All of these genes were differentially expressed, with expression fold differences ranging from 1.5 to 7.6 -fold. The HSP40 genes were more highly expressed in degenerate embryos, whereas the HSP70 members were more highly expressed in blastocysts. Thus, expression and function of these genes may play an important role in mediating stress for successful development. Interestingly, the expression of these genes was found to be relevant to embryonic development when oocytes came from different sources (Pretheeban et al., 2009). In this study, parity and poor embryo quality were tested for their possible contributions to the lower pregnancy rates observed in 
mature cows compared with heifers. Semiquantitative PCR results revealed that HSP70 was more highly expressed in heifer embryos than in embryos from mature multiparous cows. Collectively, the results from these studies indicate the extent of the influence that HSP genes have on the success of a developing embryo, with expression of HSP70 potentially underlying successful blastocyst development.

It is well documented that in vivo embryos have a better development outcome compared with in vitro-derived embryos because of alterations in gene expression. Following embryo transfer to a recipient, about $13 \%$ of in vitro-derived embryos will be aborted, whereas the abortion rate is about $5 \%$ with in vivo-derived embryos (Farin et al., 2006). It is believed that the stresses that an in vitro culture system places on an embryo may perturb development. Many studies have analyzed the relative gene expression of developmentally important genes in response to and throughout the manipulations of the IVF process (Wrenzycki et al., 2001; Knijn et al., 2002; Rizos et al., 2002b; Lonergan et al., 2003; Tesfaye et al., 2004; Knijn et al., 2005; Gad et al., 2012). In Knijn et al. (2002), results suggested that gene expression within blastocysts of oocytes collected at pre- and post-LH surge and then matured in vitro was not significantly different. Thus, in vitro blastocyst mRNA expression is not affected by time of collection of the oocytes, and gene expression changes could be in response to other components of the IVF process. These findings are in concordance with those of Rizos et al. (2002c), where the quality of the oocyte seems to affect overall blastocyst yield, whereas the culture environment affects blastocyst quality.

Wrenzycki et al. (2001) tested gene expression of in vitro embryos cultured in various media and supplements compared with in vivo-derived embryos. Evidence from this study, as well as from Tesfaye et al. (2004), suggests that the in vitro production environment alters the expression of developmentally critical genes compared with in vivo-derived embryos. Similarly, a study by Kepkova et al. (2011) identified a panel of 5 genes differentially expressed in the late 8-cell stage between in vivo- and in vitro-derived embryos. The genes differentially expressed in the latter study largely modulate transcription; thus, differential expression of genes as a result of production systems may yield different development potential and should continue to be studied for improvement of in vitro production systems.

To better elucidate how embryos from different production methods differ in genetic makeup, RNA-Seq was used to profile the transcriptome of both in vivo and in vitro embryo populations (Driver et al., 2012). Interestingly, 793 genes showed differential expression between in vitro- and in vivo-derived embryos, with
35 genes uniquely expressed within the in vivo embryos and 10 genes uniquely expressed within the in vitro embryos. Pathway enrichment analysis showed that 11 genes of the cholesterol biosynthesis pathway were more highly expressed in the in vitro embryos. Additionally, 873 genes showed differential alternative splicing between groups, and 45 novel transcribed units were found to be differentially expressed. As RNA-Seq is a novel technology, the study exhibits the power of detection of lowly expressed transcripts and further annotation of embryonic transcripts within the bovine reference genome including transcript variants.

Although the study by Driver et al. (2012) identified unannotated bovine embryonic transcripts, a comparative approach across mammalian model organisms may also be helpful to identify the functional role of novel transcribed units (NTU) that were previously unannotated in the bovine reference genome. Zhang et al. (2012) analyzed NTU and alternatively spliced transcripts identified by RNA-Seq within a population of blastocysts and degenerate embryos to better understand their function. Of the NTU, 8 were tested and found to have 2.5- to 90-fold differential expression between blastocysts and degenerates. Additionally, sequence homology of the NTU was similar between cattle and human; therefore, comparatively, the roles and functions may be inferred across species. Significantly expressed genes included an imprinted gene (ZIM3), a transcription factor $(S O X 9)$, and a gene that mediates protein-protein interactions (with homology to bovine F-box protein-like gene). Moreover, 5 of the alternatively spliced transcripts showed marked differential expression between blastocyst and degenerate embryo populations, suggesting that expression of certain transcripts may be indicative of blastocyst formation and development.

As observed in the previously described study, the identification of new, unannotated transcripts within the embryo limits the capability of microarrays generated for bovine applications. Recently, the EmbryoGENE Bovine Microarray (http://embryogene.ca/ EmbryoGENE_transcriptomics_platform) has been designed to allow for expression analysis of embryospecific transcripts for a large number of samples (Robert et al., 2011). Incorporated into the design of the microarray are 42,242 probes, which include known reference genes, novel transcribed regions taken from deep sequencing data, alternatively spliced products, probes for detection of $3^{\prime}$ untranslated region (UTR) variants, and control probes. Application of this technology was reported in a recent study by Gad et al. (2012), which analyzed the transcripts of blastocysts derived from in vitro and in vivo conditions and the extent to which culture conditions affect embryonic genome 
activation. Genes differentially expressed were involved in pathways relating to lipid metabolism and oxidative stress. These findings demonstrate the sensitivity of the embryo transcriptome to culture environment at the time of EGA, a developmentally critical window. Studies correlating these in vitro changes to pregnancy outcome are currently not available.

\section{EPIGENETICS OF FERTILITY AND DEVELOPMENT}

\section{Maternal Epigenetics}

Imprinted genes are expressed in a parent-of-origin manner, and it may be crucial to better understand their part in changes in the epigenetic profile within the oocyte. It has been found in oocytes that, irrespective of follicle size, histone 3 was dimethylated at position $\mathrm{k} 9$, which is associated with inactive genes, and histone 4 was acetylated at position $\mathrm{k} 12$, which is associated with active genes (Racedo et al., 2009). Although Racedo et al. (2009) found that the acetylation of histone 4 changed during maturation, both epigenetic marks were relatively similar for oocytes derived from varying follicle size. It was also observed that although the previous mentioned epigenetic marks do not vary with follicle size, relative abundance of gene transcripts thought to contribute to reprogramming, such as DNA methyltransferase 1 (DNMT1), were found to be more abundant in oocytes isolated from follicles of 2 to 8 mm (Racedo et al., 2009). Furthermore, O'Doherty et al. (2012) compared methylation levels across bovine oocytes of different growth stages. Those oocytes of size $\geq 120 \mu \mathrm{m}$ had a greater degree of methylation across the differentially methylated regions for 3 imprinted genes, PLAGL1, PEG10, and SNRPN, compared with oocytes of 101 to $110 \mu \mathrm{m}$. As DNA methyltransferases facilitate methylation, detection of their presence was warranted and confirmed by reverse transcription-PCR and Western blot analysis; DNMT3a, DNMT3b, and DNMT3L were expressed throughout the growth of the oocyte. Therefore, the stage of growth of the oocyte corresponds to the degree of imprinting status.

As the media used in an in vitro system may alter the expression of maternal mRNA transcripts, some studies have particularly focused on the alterations of imprinted genes within different production environments. Research by Katz-Jaffe et al. (2009) compared the gene expression of putatively imprinted genes by microarray in in vivo- and in vitro-matured bovine oocytes. It was found that the genes IGF2R, PEG3, and $S N R P N$ were upregulated in the in vitro-matured oocyte population, suggesting that culture environment may affect the regulatory mechanisms of these genes, resulting in aberrant expression. Similarly, Heinzmann et al. (2011) studied the effects of 2 in vitro maturation systems compared with an in vivo maturation system on the expression of bovine imprinted genes. Within the in vitro maturation systems, expression of the genes H19, $I G F 2 R$, and PEG3 were all upregulated in contrast to in vivo-matured oocytes, and no significant differences were observed between immature oocytes and in vitromatured oocytes. Interestingly, upregulation of the methyltransferases DNMT1a, DNMT 3a, and DNMT $3 b$ was observed in both in vitro maturation systems compared with in vivo-matured oocytes. Although the expression of the methyltransferases was upregulated within in vitro production systems, the percentage of aberrantly methylated $\mathrm{CpG}$ islands within the cisregulatory regions of the genes was not statistically different. Thus, culture conditions may influence the expression of imprinted genes; however, further analysis is needed to better understand the causative mechanisms underlying this aberrant expression.

\section{Paternal Epigenetics}

Genetic remodeling is well documented over the course of mammalian spermatogenesis (Miller et al., 2005). Histone modifications that occur during spermatogenesis provide a mechanism for epigenetic modification (Carrell, 2012). During spermatogenesis, histones are incompletely replaced with temporary transition proteins. These temporary proteins are subsequently replaced by protamines to form a torroid structure. This structure results in highly condensed DNA, thereby shutting down transcription. Incomplete histone to protamine exchange often occurs in the promoter region of genes that are important in embryonic development (Jenkins and Carrell, 2012). Errors in histone exchange may lead to incorrect chromatin repackaging, which is associated with decreased fertility in humans (Miller et al., 2010). At the time of fertilization, the paternal genome is again remodeled through H4 hyperacetylation (Mason et al., 2012). Remodeling of the paternal genome could therefore largely contribute to differential expression of developmentally important genes and delivery of paternal transcripts at the time of fertilization.

Other epigenetic effects associated with fertility include methylation and associated imprinted gene expression. In human fertility cases, aberrant methylation of specific genes (e.g., DAZL and MTHFR) and other relevant genes, such as imprinted genes, are strongly associated with infertility and sperm defects (as reviewed by Jenkins and Carrell, 2012). Also, global sperm hypomethylation has been found to be associated with poor pregnancy outcomes in human IVF patients. Further analysis of the characterization of methylation marks 
of bovine spermatozoa as it contributes to fertilization should be a goal for future research.

At the time of fertilization, a total sperm "package" is delivered to the oocyte. It is crucial to the early zygote that many of these epigenetic processes occur as the products under epigenetic regulation are delivered to the oocyte. Both the ratio of specific protamines and the histone exchange for protamines at appropriate sites dictate which genes may be transcribed, because DNA bound by histones is less condensed and therefore available for transcription. Moreover, key regions of histone retention include imprinted genes, miRNA clusters, and key developmental gene promoters (Jenkins and Carrell, 2012). At the genomic level, histones can facilitate histone tail modifications such as methylation, phosphorylation, or acetylation to activate or suppress expression of genes (Jenkins and Carrell, 2012). At the time of fertilization, gene products within the sperm package include transcription factors, signaling molecules, sperm-specific transcripts, and miRNA (Krawetz, 2005). These products within the package make up the paternal contribution, which is regulated by the changes made at the genomic level in structure and by further epigenetic modifications.

\section{Embryonic Epigenetics}

Evidence from studies of embryos of altered parental contribution such as parthenotes (bimaternal genome) and androgenotes (bipaternal genome) have shed light on the importance of parent-of-origin expression of both maternal and paternal alleles of imprinted genes. Imprinted genes are regulated by epigenetic modifications such as genomic methylation, histone modification, and chromatin remodeling (Kono, 2009). The process of reprogramming the differentially methylated regions thought to regulate these genes occurs at 2 different points: at gametogenesis and following fertilization (Reik et al., 2001). By reprogramming the genome, the imprints of the parental chromosomes are erased and in each generation new imprints are made within the offspring's genome (Obata, 2011). In early embryonic development, subsequent to fertilization, the embryo's DNA undergoes demethylation followed by processes of active and passive remethylation (Reik et al., 2001). Therefore, the zygote is in state of totipotency (Golding et al., 2011; Mason et al., 2012). Then, the process of de novo remethylation that begins at the 8- to 16-cell stage in the bovine embryo will act to drive the differentiation and development of the embryo (Reik et al., 2001). Kues et al. (2008) found that transcripts upregulated in the 8-cell stage were related to molecular structuring and epigenetic modifications such as methylation, thereby further correlating po- tential epigenetic modifications mediating embryonic development.

As epigenetics is a growing area of research, a vast amount of knowledge can be gained by studying other model organisms and drawing information from bovine embryos by comparison. For instance, mice lacking the maternally expressed gene Phlda2 show placental overgrowth (Frank et al., 2002), whereas overexpression of this gene showed association with a retardation in placental growth (Salas et al., 2004). When studying a bovine embryonic transcriptome, Huang et al. (2010b) found that PHLDA2 was upregulated in degenerate bovine embryos by about 8 -fold. Recently, Driver et al. (2013) reported that 5 imprinted genes were upregulated in degenerate embryos, whereas 4 imprinted genes were upregulated in healthy blastocysts. Overall, data from these studies provide evidence that appropriate expression of imprinted genes may be crucial for progression through embryonic developmental stages.

\section{FUTURE AIMS}

Much progress has been made in identifying genes contributing to fertility. Greater emphasis should be placed on identifying genes that are conserved across mammalian species. A comparative analysis allows for identification of new genes in a species where the reference genome is less well annotated. In other organisms, the capability of gene knockdown allows for identification of genetic mutations by loss of function. A recent study in the bovine found that small interfering (si)RNA delivery into 1-cell embryos resulted in an associated increase in blastocyst development for the imprinted gene PHLDA2, whereas siRNA delivery of the imprinted gene $C D K N 1 C$ was found to decrease blastocyst development (Driver et al., 2013). Functionally, CDKN1C is a cell cycle regulator in which, upon knockdown within bovine embryos, the RNA-Seq analysis revealed differential expression of apoptosisrelated genes. Pathway analysis revealed a decline in endonuclease activity associated with cellular and DNA damage repair mechanisms and a probable mechanism for the decline in blastocyst development observed. Using pathway analysis or finding association with bovine orthologs of conserved genes with a given disease condition or development potential could provide a better understanding the functions of these genes. Conversely, if conserved genes are found, this knowledge could be used to increase knowledge of not only the bovine species but also other model organisms and humans.

Research should also focus on studying the function of a gene in both parental contributions to the embryo to better understand how these particular genes may be influenced by environment. A study by Li et al. (2012b) 
illustrated that although a certain $M A P 1 B$ maternal genotype was associated with higher fertilization and blastocyst rate, the same paternal genotype was associated with reproductive failure in females. Therefore, a comprehensive approach of investigating both maternal and paternal contributions to embryonic development should be considered. Not only are biallelically expressed genes important to study, but so, too, are parental contributions in the context of epigenetics. To a greater extent, the process of reprogramming during gametogenesis and at fertilization of these genes may greatly affect the expression of the gene; thus, further investigation is warranted. Similarly, the environment could alter reprogramming to change the parental specific expression of an allele. Research in human and mouse have revealed an association between maternal caloric intake and associated disease states later in life (Bromfield et al., 2008). It has been hypothesized by Hales and Barker (2001) that although epigenetic mechanisms may be involved, changes in the developing fetus as a response to maternal diet may serve as preparation for the environment in which the offspring must survive. As illustrated here, in vitro culture environments have the potential to cause aberrant expression; hence, finding new variations of reproductive technology that minimize changes in expression would be advantageous.

As technology has advanced, research should be refocused on not only single gene mutations but rather whole networks that include gene interactions. Recent studies by Cochran et al. (2013a,b) provide evidence that the candidate gene approach could still be a useful method for identifying genetic markers associated with reproductive traits. However, whereas microarray analysis allows for the identification of candidate genes for selection and relative expression of genes, a shift in utilizing next-generation sequencing for hypothesisgenerating research allows for greater data generation. Although an embryo-specific microarray was recently designed by Robert et al. (2011) for analysis of multiple samples of known gene probes, RNA-Seq analysis allows identification of additional genetic components. As the reference bovine genome is far from complete, sensitive, high-throughput RNA-Seq technology facilitates discovery of new transcripts, alternatively spliced transcripts, identification of lowly expressed genes, and epigenetic marks. Also, a transcriptomic approach is capable of analyzing not only the expression of single genes, but also gene interactions via pathway analysis. Enriched pathways can better elucidate how selection of single genes may affect necessary functions of a developing embryo and thereby give insight for more effective selection. This method would then allow for a greater understanding of how selection of parental genes could affect certain mechanisms. Similarly, it opens up the ability to select developmentally competent embryos for use in assisted reproductive technologies based on the expression of certain genes identified as predictors of successful development.

As genetic data are generated from a multitude of studies in this area, entry of data into large databases would open up the opportunity to share knowledge of genes and gene interactions gained by each study to further progress the field. A large criticism and limitation to in vitro studies is the lack of uniformity across production systems. Developing a data bank of experimental design or standards to standardize this system may enhance our ability to not only share but also make comparisons across data. In both in vitro and in vivo cases, it would be preferable to classify population dynamics and environmental conditions with each data set. Strides have been made to create uniformity across in vitro systems as demonstrated by Robert et al. (2011); however, further support of data banking would develop this idea further. Similarly, challenges in studying fertility in in vivo settings arise. One of these challenges is varying population dynamics, including type of animal crosses, environmental conditions, management practices, and uniform data collection. These factors are just a few that make associations or expression sometimes difficult to repeat, providing a challenge to studying fertility.

\section{CONCLUSIONS}

Over time, intense selection for milk production has had detrimental effects on fertility. Future investigation should focus on the relationship between expression and its function in driving the development of the embryo to more accurately classify those embryos that are developmentally competent. Parental genomic contributions provide the early molecular cues that drive early development and, as such, understanding these genetic prompts is essential to understanding the progression of normal development. Extensive gains in knowledge need to be made in the bovine to better understand species-specific changes in expression that are cued by the environment. As assisted reproductive technologies and dietary conditions have been shown to alter the expression of genes known to influence development, further analysis of these effects is crucial for identifying optimal in vitro systems for embryo generation and culture. Evolution of new technology has given us the tools to discover and characterize molecular mechanisms. Thus, research should focus on fully elucidating developmental mechanisms for ultimate application of that information in genetic selection in production systems and in assisted reproductive technologies for improve- 
ment in embryonic survival. Moving forward into an evolving research era, the complexity underlying the genetics of fertility and development should encompass many approaches to further our understanding. Future studies should pursue the identification of causative mutations affecting bovine fertility and embryonic lethality. As many previous studies performed GWAS to identify genes associated with these complex traits, follow-up should be performed to better characterize the role of candidate genes in the biologic mechanism. Focusing on functional studies of candidate genes by using knockout models or gene knockdowns could provide insight into their biological role. One challenge is interpreting data across studies due to the lack of a uniform experimental design. In the era of systems biology, a study design that encompasses an "omics" approach would help us to better understand cues driving these biologic mechanisms. A great deal of data could be generated from this approach and may help in bridging the unknown between each parental contribution leading up to fertilization and the subsequent hurdles in mammalian development.

\section{ACKNOWLEDGMENTS}

This study was supported by a USDA Hatch Act formula fund 142-PRJ52YY from the College of Agriculture and Life Sciences (University of Wisconsin, Madison), and by a Graduate School Fall Competition Grant (University of Wisconsin, Madison) to SMS.

\section{REFERENCES}

Abd El Naby, W. S., T. H. Hagos, M. M. Hossain, D. Salilew-Wondim, A. Y. Gad, F. Rings, M. U. Cinar, E. Tholen, C. Looft, K. Schellander, M. Hoelker, and D. Tesfaye. 2013. Expression analysis of regulatory microRNAs in bovine cumulus oocyte complex and preimplantation embryos. Zygote 21:31-51.

Arangasamy, A., V. R. Kasimanickam, J. M. DeJarnette, and R. K. Kasimanickam. 2011. Association of CRISP2, CCT8, PEBP1 mRNA abundance in sperm and sire conception rate in Holstein bulls. Theriogenology 76:570-577.

Berry, D. P., J. W. M. Bastiaansen, R. F. Veerkamp, S. Wijga, E. Wall, B. Berglund, and M. P. L. Calus. 2012. Genome-wide associations for fertility traits in Holstein-Friesian dairy cows using data from experimental research herds in four European countries. Animal 6:1206-1215

Blaschek, M., A. Kaya, N. Zwald, E. Memili, and B. W. Kirkpatrick. 2011. A whole-genome association analysis of noncompensatory fertility in Holstein bulls. J. Dairy Sci. 94:4695-4699.

Braundmeier, A. G., and D. J. Miller. 2001. The search is on: Finding accurate molecular markers of male fertility. J. Dairy Sci. 84:1915-1925.

Bressan, F. F., T. H. C. De Bem, F. Perecin, F. L. Lopes, C. E. Ambrosio, F. V. Meirelles, and M. A. Miglino. 2009. Unearthing the roles of imprinted genes in the placenta. Placenta 30:823-834.

Bromfield, J., W. Messamore, and D. F. Albertini. 2008. Epigenetic regulation during mammalian oogenesis. Reprod. Fertil. Dev. 20:74-80.

Card, C. J., E. J. Anderson, S. Zamberlan, K. E. Krieger, M. Kaproth, and B. L. Sartini. 2013. Cryopreserved bovine spermato- zoal transcript profile as revealed by high-throughput ribonucleic acid sequencing. Biol. Reprod. 88:49.http://dx.doi.org/10.1095/ biolreprod.112.103788.

Carrell, D. T. 2012. Epigenetics of the male gamete. Fertil. Steril. 97:267-274

Cochran, S. D., J. B. Cole, D. J. Null, and P. J. Hansen. 2013a. Discovery of single nucleotide polymorphisms in candidate genes associated with fertility and production traits in Holstein cattle. BMC Genet. 14:49

Cochran, S. D., J. B. Cole, D. J. Null, and P. J. Hansen. 2013b. Single nucleotide polymorphisms in candidate genes associated with fertilizing ability of sperm and subsequent embryonic development in cattle. Biol. Reprod. 89:69. http://dx.doi.org/10.1095/ biolreprod.113.111260.

Cole, J. B., G. R. Wiggans, L. Ma, T. S. Sonstegard, T. J. Lawlor Jr., B. A. Crooker, C. P. Van Tassell, J. Yang, S. Wang, L. K. Matukumalli, and Y. Da. 2011. Genome-wide association analysis of thirty one production, health, reproduction and body conformation traits in contemporary US Holstein cows. BMC Genomics 12:408.

Crosier, A. E., P. W. Farin, M. J. Dykstra, J. E. Alexander, and C. E. Farin. 2001. Ultrastructural morphometry of bovine blastocysts produced in vivo or in vitro. Biol. Reprod. 64:1375-1385.

DeJarnette, J. M., C. E. Marshall, R. W. Lenz, D. R. Monke, W. H. Ayars, and C. G. Sattler. 2004. Sustaining the fertility of artificially inseminated dairy cattle: The role of the artificial insemination industry. J. Dairy Sci. 87(E-Suppl.):E93-E104.

Diskin, M. G., M. H. Parr, and D. G. Morris. 2011. Embryo death in cattle: An update. Reprod. Fertil. Dev. 24:244-251.

Driver, A. M., W. Huang, S. Gajic, R. L. Monson, G. J. M. Rosa, and H. Khatib. 2009. Short communication: Effects of the progesterone receptor variants on fertility traits in cattle. J. Dairy Sci. 92:4082-4085.

Driver, A. M., W. Huang, J. Kropp, F. Peñagaricano, and H. Khatib. 2013. Knockdown of $C D K N 1 C\left(p 5^{\text {tip } 2}\right)$ and PHLDA2 results in developmental changes in bovine pre-implantation embryos. PLoS ONE 8:e69490. http://dx.doi.org/10.1371/journal.pone.0069490.

Driver, A. M., and H. Khatib. 2013. Physiology and Endocrinology Symposium: Heat shock proteins: Potentially powerful markers for preimplantation embryonic development and fertility in livestock species. J. Anim. Sci. 91:1154-1161.

Driver, A. M., F. Peñagaricano, W. Huang, K. R. Ahmad, K. S. Hackbart, M. C. Wiltbank, and H. Khatib. 2012. RNA-Seq analysis uncovers transcriptomic variations between morphologically similar in vivo- and in vitro-derived bovine blastocysts. BMC Genomics $13: 118$

Evans, A. C. O., and S. W. Walsh. 2011. The physiology of multifactorial problems limiting the establishment of pregnancy in dairy cattle. Reprod. Fertil. Dev. 24:233-237.

Farin, P. W., J. A. Piedrahita, and C. E. Farin. 2006. Errors in development of fetuses and placentas from in vitro-produced bovine embryos. Theriogenology 65:178-191.

Feugang, J. M., A. Kaya, G. P. Page, L. Chen, T. Mehta, K. Hirani, L. Nazareth, E. Topper, R. Gibbs, and E. Memili. 2009. Two-stage genome-wide association study identifies integrin beta 5 as having potential role in bull fertility. BMC Genomics 10:176.

Fortes, M. R. S., W. M. Snelling, A. Reverter, S. H. Nagaraj, S. A Lehnert, R. J. Hawken, K. L. DeAtley, S. O. Peters, G. A. Silver, G. Rincon, J. F. Medrano, A. Islas-Trejo, and M. G. Thomas. 2012. Gene network analyses of first service conception in Brangus heifers: Use of genome and trait associations, hypothalamictranscriptome information, and transcription factors. J. Anim. Sci. 90:2894-2906

Frank, D., W. Fortino, L. Clark, R. Musalo, W. X. Wang, A. Saxena, C. M. Li, W. Reik, T. Ludwig, and B. Tycko. 2002. Placental overgrowth in mice lacking the imprinted gene $l p l$. Proc. Natl. Acad. Sci. USA 99:7490-7495.

Gad, A., M. Hoelker, U. Besenfelder, V. Havlicek, U. Cinar, F. Rings, E. Held, I. Dufort, M. A. Sirard, K. Schellander, and D. Tesfaye. 2012. Molecular mechanisms and pathways involved in bovine embryonic genome activation and their regulation by alternative in 
vivo and in vitro culture conditions. Biol. Reprod. 87:100. http:// dx.doi.org/10.1095/biolreprod.112.099697.

Golding, M. C., G. L. Williamson, T. K. Stroud, M. E. Westhusin, and C. R. Long. 2011. Examination of DNA methyltransferase expression in cloned embryos reveals an essential role for Dnmt1 in bovine development. Mol. Reprod. Dev. 78:306-317.

Govindaraju, A., S. Dogan, N. Rodriguez-Osorio, K. Grant, A. Kaya, and E. Memili. 2012. Delivering value from sperm proteomics for fertility. Cell Tissue Res. 349:783-793.

Hales, C. N., and D. Barker. 2001. The thrifty phenotype hypothesis. Br. Med. Bull. 60:5-20.

Heinzmann, J., T. Hansmann, D. Herrmann, C. Wrenzycki, U. Zechner, T. Haaf, and H. Niemann. 2011. Epigenetic profile of developmentally important genes in bovine oocytes. Mol. Reprod. Dev. $78: 188-201$.

Huang, W., B. W. Kirkpatrick, G. J. M. Rosa, and H. Khatib. 2010a. A genome-wide association study using selective DNA pooling identifies candidate markers for fertility in Holstein cattle. Anim. Genet. 41:570-578.

Huang, W., B. S. Yandell, and H. Khatib. 2010b. Transcriptomic profiling of bovine IVF embryos revealed candidate genes and pathways involved in early embryonic development. BMC Genomics $11: 23$

Jenkins, T. G., and D. T. Carrell. 2012. The sperm epigenome and potential implications for the developing embryo. Reproduction 143:727-734

Kasimanickam, V., R. Kasimanickam, A. Arangasamy, A. Saberivand, J. S. Stevenson, and J. P. Kastelic. 2012. Association between mRNA abundance of functional sperm function proteins and fertility of Holstein bulls. Theriogenology 78:2007-2019

Katz-Jaffe, M. G., B. R. McCallie, K. A. Preis, J. Filipovits, and D. K. Gardner. 2009. Transcriptome analysis of in vivo and in vitro matured bovine MII oocytes. Theriogenology 71:939-946.

Kepkova, K. V., P. Vodicka, T. Toralova, M. Lopatarova, S. Cech, R. Dolezel, V. Havlicek, U. Besenfelder, A. Kuzmany, M. A. Sirard, J. Laurincik, and J. Kanka. 2011. Transcriptomic analysis of in vivo and in vitro produced bovine embryos revealed a developmental change in cullin 1 expression during maternal-to-embryonic transition. Theriogenology 75:1582-1595.

Khatib, H., W. Huang, X. Wang, A. H. Tran, A. B. Bindrim, V. Schutzkus, R. L. Monson, and B. S. Yandell. 2009. Single gene and gene interaction effects on fertilization and embryonic survival rates in cattle. J. Dairy Sci. 92:2238-2247.

Khatib, H., C. Maltecca, R. L. Monson, V. Schutzkus, X. Wang, and J. J. Rutledge. 2008a. The fibroblast growth factor 2 gene is associated with embryonic mortality in cattle. J. Anim. Sci 86:2063-2067.

Khatib, H., R. L. Monson, W. Huang, R. Khatib, V. Schutzkus, H Khateeb, and J. J. Parrish. 2010. Validation of in vitro fertility genes in a Holstein bull population. J. Dairy Sci. 93:2244-2249.

Khatib, H., R. L. Monson, V. Schutzkus, D. M. Kohl, G. J. M. Rosa, and J. J. Rutledge. 2008b. Mutations in the STAT5A gene are associated with embryonic survival and milk composition in cattle. J. Dairy Sci. 91:784-793.

Knijn, H. M., C. Wrenzycki, P. J. M. Hendriksen, P. Vos, D. Herrmann, G. C. van der Weijden, H. Niemann, and S. J. Dieleman. 2002. Effects of oocyte maturation regimen on the relative abundance of gene transcripts in bovine blastocysts derived in vitro or in vivo. Reproduction 124:365-375.

Knijn, H. M., C. Wrenzycki, P. J. M. Hendriksen, P. Vos, E. C. Zeinstra, G. C. van der Weijden, H. Niemann, and S. J. Dieleman. 2005. In vitro and in vivo culture effects on mRNA expression of genes involved in metabolism and apoptosis in bovine embryos. Reprod. Fertil. Dev. 17:775-784.

Kono, T. 2009. Genetic modification for bimaternal embryo development. Reprod. Fertil. Dev. 21:31-36.

Krawetz, S. A. 2005. Paternal contribution: New insights and future challenges. Nat. Rev. Genet. 6:633-642.

Kues, W. A., S. Sudheer, D. Herrmann, J. W. Carnwath, V. Havlicek, U. Besenfelder, H. Lehrach, J. Adjaye, and H. Niemann. 2008. Genome-wide expression profiling reveals distinct clusters of tran- scriptional regulation during bovine preimplantation development in vivo. Proc. Natl. Acad. Sci. USA 105:19768-19773.

Lalancette, C., C. Thibault, I. Bachand, N. Caron, and N. Bissonnette. 2008. Transcriptome analysis of bull semen with extreme nonreturn rate: Use of suppression-subtractive hybridization to identify functional markers for fertility. Biol. Reprod. 78:618-635.

Lan, X. Y., F. Peñagaricano, L. DeJung, K. A. Weigel, and H. Khatib. 2013. A missense mutation in the PROP1 (prophet of Pit 1) gene affects male fertility and milk production traits in the US Holstein population. J. Dairy Sci. 96:1255-1257.

Laporta, J., A. Driver, and H. Khatib. 2011. Short communication: Expression and alternative splicing of POU1F1 pathway genes in preimplantation bovine embryos. J. Dairy Sci. 94:4220-4223.

Leroy, J. L., G. Opsomer, A. Van Soom, I. G. F. Goovaerts, and P. Bols. 2008. Reduced fertility in high-yielding dairy cows: Are the oocyte and embryo in danger? Part I: The importance of negative energy balance and altered corpus luteum function to the reduction of oocyte and embryo quality in high-yielding dairy cows. Reprod. Domest. Anim. 43:612-622.

Li, G., K. Khateeb, E. Schaeffer, B. Zhang, and H. Khatib. 2012a. Genes of the transforming growth factor-beta signalling pathway are associated with pre-implantation embryonic development in cattle. J. Dairy Res. 79:310-317.

Li, G., F. Peñagaricano, K. A. Weigel, Y. Zhang, G. J. M. Rosa and H. Khatib. 2012b. Comparative genomics between fly, mouse, and cattle identifies genes associated with sire conception rate. J Dairy Sci. 95:6122-6129.

Lonergan, P., D. Rizos, A. Gutierrez-Adan, T. Fair, and M. P. Boland. 2003. Oocyte and embryo quality: Effect of origin, culture conditions and gene expression patterns. Reprod. Domest. Anim. $38: 259-267$

Lucy, M. C. 2001. ADSA Foundation Scholar Award: Reproductive loss in high-producing dairy cattle: Where will it end? J. Dairy Sci. 84:1277-1293.

Mamo, S., J. P. Mehta, P. McGettigan, T. Fair, T. E. Spencer, F. W. Bazer, and P. Lonergan. 2011. RNA sequencing reveals novel gene clusters in bovine conceptuses associated with maternal recognition of pregnancy and implantation. Biol. Reprod. 85:1143-1151.

Mason, K., Z. Liu, T. Aguirre-Lavin, and N. Beaujean. 2012. Chromatin and epigenetic modifications during early mammalian development. Anim. Reprod. Sci. 134:45-55.

Memili, E., and N. L. First. 2000. Zygotic and embryonic gene expression in cow: A review of timing and mechanisms of early gene expression as compared with other species. Zygote 8:87-96

Miller, D., M. Brinkworth, and D. Iles. 2010. Paternal DNA packaging in spermatozoa: More than the sum of its parts? DNA, histones, protamines and epigenetics. Reproduction 139:287-301.

Miller, D., G. C. Ostermeier, and S. A. Krawetz. 2005. The controversy, potential and roles of spermatozoal RNA. Trends Mol. Med. $11: 156-163$

Misirlioglu, M., G. P. Page, H. Sagirkaya, A. Kaya, J. J. Parrish, N. L. First, and E. Memili. 2006. Dynamics of global transcriptome in bovine matured oocytes and preimplantation embryos. Proc. Natl. Acad. Sci. USA 103:18905-18910.

Moore, K., and W. Thatcher. 2006. Major advances associated with reproduction in dairy cattle. J. Dairy Sci. 89:1254-1266.

O'Doherty, A. M., L. C. O'Shea, and T. Fair. 2012. Bovine DNA methylation imprints are established in an oocyte size-specific manner, which are coordinated with the expression of the DNMT3 family proteins. Biol. Reprod. 86:67.

Obata, Y. 2011. Study on the mechanism of maternal imprinting during oocyte growth. J. Reprod. Dev. 57:1-8.

Ogorevc, J., P. Dovc, and T. Kunej. 2011. Comparative genomics approach to identify candidate genetic loci for male fertility. Reprod. Domest. Anim. 46:229-239.

Ostermeier, G. C., D. Miller, J. D. Huntriss, M. P. Diamond, and S. A. Krawetz. 2004. Reproductive biology-Delivering spermatozoan RNA to the oocyte. Nature 429:154.

Peddinti, D., B. Nanduri, A. Kaya, J. M. Feugang, S. C. Burgess, and E. Memili. 2008. Comprehensive proteomic analysis of bovine sper- 
matozoa of varying fertility rates and identification of biomarkers associated with fertility. BMC Syst. Biol. 2:19.

Peñagaricano, F., and H. Khatib. 2012. Association of milk protein genes with fertilization rate and early embryonic development in Holstein dairy cattle. J. Dairy Res. 79:47-52.

Peñagaricano, F., K. A. Weigel, and H. Khatib. 2012a. Genome-wide association study identifies candidate markers for bull fertility in Holstein dairy cattle. Anim. Genet. 43:65-71.

Peñagaricano, F., K. A. Weigel, G. J. M. Rosa, and H. Khatib. 2012b. Inferring quantitative trait pathways associated with bull fertility from a genome-wide association study. Front. Genet. 3:307.

Pretheeban, T., M. Gordon, R. Singh, R. Perera, and R. Rajamahendran. 2009. Differential mRNA expression in in vivo produced pre-implantation embryos of dairy heifers and mature cows. Mol. Reprod. Dev. 76:1165-1172.

Racedo, S. E., C. Wrenzycki, D. Herrmann, D. Salamone, and H. Niemann. 2008. Effects of follicle size and stages of maturation on mRNA expression in bovine in vitro matured oocytes. Mol. Reprod. Dev. 75:17-25.

Racedo, S. E. C. Wrenzycki, K. Lepikhov, D. Salamone, J. Walter and H. Niemann. 2009. Epigenetic modifications and related mRNA expression during bovine oocyte in vitro maturation. Reprod. Fertil. Dev. 21:738-748.

Reik, W., W. Dean, and J. Walter. 2001. Epigenetic reprogramming in mammalian development. Science 293:1089-1093.

Rizos, D., T. Fair, S. Papadopoulos, M. P. Boland, and P. Lonergan. 2002a. Developmental, qualitative, and ultrastructural differences between ovine and bovine embryos produced in vivo or in vitro. Mol. Reprod. Dev. 62:320-327.

Rizos, D., P. Lonergan, M. P. Boland, R. Arroyo-Garcia, B. Pintado, J. de la Fuente, and A. Gutierrez-Adan. 2002b. Analysis of differential messenger RNA expression between bovine blastocysts produced in different culture systems: Implications for blastocyst quality. Biol. Reprod. 66:589-595.

Rizos, D., F. Ward, P. Duffy, M. P. Boland, and P. Lonergan. 2002c. Consequences of bovine oocyte maturation, fertilization or early embryo development in vitro versus in vivo: Implications for blastocyst yield and blastocyst quality. Mol. Reprod. Dev. 61:234248

Robert, C., J. Nieminen, I. Dufort, D. Gagne, J. R. Grant, G. Cagnone, D. Plourde, A. L. Nivet, E. Fournier, E. Paquet, M. Blazejczyk, P. Rigault, N. Juge, and M. A. Sirard. 2011. Combining resources to obtain a comprehensive survey of the bovine embryo transcriptome through deep sequencing and microarrays. Mol. Reprod. Dev. 78:651-664.
Russell, D. F., S. Baqir, J. Bordignon, and D. H. Betts. 2006. The impact of oocyte maturation media on early bovine embryonic development. Mol. Reprod. Dev. 73:1255-1270.

Sahana, G., B. Guldbrandtsen, C. Bendixen, and M. S. Lund. 2010. Genome-wide association mapping for female fertility traits in Danish and Swedish Holstein cattle. Anim. Genet. 41:579-588.

Salas, M., R. John, A. Saxena, S. Barton, D. Frank, G. Fitzpatrick, M. J. Higgins, and B. Tycko. 2004. Placental growth retardation due to loss of imprinting of Phlda2. Mech. Dev. 121:1199-1210. PubMed

Santos, J. E. P., W. W. Thatcher, R. C. Chebel, R. L. A. Cerri, and K. N. Galvao. 2004. The effect of embryonic death rates in cattle on the efficacy of estrus synchronization programs. Anim. Reprod. Sci. 82-83:513-535.

Sirard, M. A., F. Richard, P. Blondin, and C. Robert. 2006. Contribution of the oocyte to embryo quality. Theriogenology 65:126-136

Tesfaye, D., S. Ponsuksili, K. Wimmers, M. Gilles, and K. Schellander. 2004. A comparative expression analysis of gene transcripts in post-fertilization developmental stages of bovine embryos produced in vitro or in vivo. Reprod. Domest. Anim. 39:396-404.

Tesfaye, D., D. Worku, F. Rings, C. Phatsara, E. Tholen, K. Schellander, and M. Hoelker. 2009. Identification and expression profiling of microRNAs during bovine oocyte maturation using heterologous approach. Mol. Reprod. Dev. 76:665-677.

Van Soom, A., B. Mateusen, J. Leroy, and A. De Kruif. 2003. Assessment of mammalian embryo quality: What can we learn from embryo morphology? Reprod. Biomed. Online 7:664-670. PubMed.

Veerkamp, R. F., and B. Beerda. 2007. Genetics and genomics to improve fertility in high producing dairy cows. Theriogenology 68:S266-S273

Walsh, S. W., E. J. Williams, and A. C. O. Evans. 2011. A review of the causes of poor fertility in high milk producing dairy cows. Anim. Reprod. Sci. 123:127-138.

Wrenzycki, C., D. Herrmann, L. Keskintepe, A. Martins, S. Sirisathien, B. Brackett, and H. Niemann. 2001. Effects of culture system and protein supplementation on mRNA expression in pre-implantation bovine embryos. Hum. Reprod. 16:893-901.

Zhang, B., F. Peñagaricano, H. Chen, and H. Khatib. 2012. Novel transcripts and alternatively spliced genes are associated with early development in bovine embryos. Animal 6:1199-1205.

Zhang, B., F. Peñagaricano, A. Driver, H. Chen, and H. Khatib. 2011. Differential expression of heat shock protein genes and their splice variants in bovine preimplantation embryos. J. Dairy Sci. 94:4174-4182. 